\title{
Eurostudia
}

\section{Les morts littéraires de Jean de Brébeuf : l'histoire coloniale et l'imaginaire canadien}

Paul D. Morris

Volume 13, numéro 1-2, 2018-2019

Cultures en contact, entre régulations et représentations

URI : https://id.erudit.org/iderudit/1067287ar

DOI : https://doi.org/10.7202/1067287ar

Aller au sommaire du numéro

Éditeur(s)

Le Centre canadien d'études allemandes et européennes

ISSN

1718-8946 (numérique)

Découvrir la revue

Citer cet article

Morris, P. D. (2018). Les morts littéraires de Jean de Brébeuf : l'histoire coloniale et l'imaginaire canadien. Eurostudia, 13(1-2), 345-368. https://doi.org/10.7202/1067287ar
Résumé de l'article

La vie et surtout la mort du missionnaire jésuite Jean de Brébeuf en Huronie au $\mathrm{XVII}^{\mathrm{e}}$ siècle occupent une place complexe et évolutive dans l'imaginaire canadien. Un personnage clé au moment du contact initial entre les colonisateurs européens et les peuples indigènes de l'Amérique du Nord, Brébeuf fut destiné à devenir encore plus grand que le rôle historique qu'il a joué dans sa vie. Avec sa mort, Brébeuf entra dans le domaine des représentations mythiques du Canada. L'article actuel cherche à tracer la trajectoire historique de la place de Brébeuf dans les littératures d'expression anglophone et francophone au Canada. Faisant référence à une lecture de trois textes littéraires représentatifs, l'article suggère que la représentation littéraire de Brébeuf peut être étroitement alignée sur des conceptions historiquement changeantes du Canada selon des compréhensions francophone, anglophone et autochtone de l'identité nationale.
Tous droits réservés $@$ Le Centre canadien d'études allemandes et européennes, 2019
Ce document est protégé par la loi sur le droit d'auteur. L'utilisation des services d’Érudit (y compris la reproduction) est assujettie à sa politique d'utilisation que vous pouvez consulter en ligne.

https://apropos.erudit.org/fr/usagers/politique-dutilisation/ 
Les morts littéraires de Jean de Brébeuf:

l'histoire coloniale et l'imaginaire canadien

\author{
Paul D. Morris \\ Université de Saint-Boniface
}

\title{
Résumé
}

La vie et surtout la mort du missionnaire jésuite Jean de Brébeuf en Huronie au XVII e siècle occupent une place complexe et évolutive dans l'imaginaire canadien. Un personnage clé au moment du contact initial entre les colonisateurs européens et les peuples indigènes de l'Amérique du Nord, Brébeuf fut destiné à devenir encore plus grand que le rôle historique qu'il a joué dans sa vie. Avec sa mort, Brébeuf entra dans le domaine des représentations mythiques du Canada. L'article actuel cherche à tracer la trajectoire historique de la place de Brébeuf dans les littératures d'expression anglophone et francophone au Canada. Faisant référence à une lecture de trois textes littéraires représentatifs, l'article suggère que la représentation littéraire de Brébeuf peut être étroitement alignée sur des conceptions historiquement changeantes du Canada selon des compréhensions francophone, anglophone et autochtone de l'identité nationale.

\section{Abstract}

The life, and above all the death, of the Jesuit missionary Jean de Brébeuf in $17^{\text {th }}$ century Huronia occupies a complex and evolving position in the Canadian imaginary. A key historical figure at the time of initial contact between European colonisers and the Indigenous peoples of what is now Canada, Brébeuf was destined to become larger than the historical role he played in 
life. With his death, Brébeuf entered into the realm of mythic representations of Canada. The present article seeks to trace the historical trajectory of Brébeuf's place in anglophone and francophone Canadian literature. Through a reading of three representative literary texts, the article will suggest that the literary depiction of Brébeuf may be closely aligned with historically changing conceptions of Canada according to Francophone, Anglophone and Indigenous understandings of the national identity.

Dans sa nouvelle de 1966 intitulée «The Loons », l'écrivaine canadienne Margaret Laurence présente Vanessa, une fille intellectuellement précoce de 11 ans, se débattant avec les émotions provoquées par la présence inattendue dans sa vie d'une jeune fille métisse, Piquette Tonnerre. Malgré la présence d'une communauté métisse qui habite un campement à la périphérie de son village manitobain, Vanessa, la narratrice, se rend compte qu'en fait, elle n'a aucune idée de l'expérience vécue de Piquette. Afin de comprendre la spécificité troublante de l'identité indigène de la personne qui se tient devant elle, Vanessa s'en remet à un ensemble de détails contingents portant sur diverses figures historiques autochtones :

In the days that followed, however, Piquette began to interest me, and I began to want to interest her. [...] Unlikely as it may seem, I had only just realized that the Tonnerre family, whom I had always heard called half-breed, were actually Indians, or as near as made no difference. My acquaintance with Indians was not extensive. I did not remember ever having seen a real Indian, and my new awareness that Piquette sprang from the people of Big Bear and Poundmaker, of Tecumsah, of the Iroquois who had eaten Father Brebeuf's heart - all of this gave her an instant attraction in my eyes (Laurence $1993: 112)^{1}$.

\footnotetext{
1 «Cependant, dans les jours qui ont suivi, Piquette a commencé à m'intéresser et j'ai commencé à vouloir l'intéresser. [...] Aussi improbable que cela puisse paraître, je venais tout juste de me rendre compte que la famille Tonnerre, qui m'avait toujours été présentée comme métisse, était en réalité indienne ou à peu
} 
Même si l'ignorance relative de Vanessa joue un rôle d'importance dans la nouvelle, dans le contexte présent c'est plutôt la nature de ses connaissances limitées qui retient l'attention. L'inventaire des images autochtones évoquées par Vanessa est axé sur des figures historiques au Canada et inclut Jean de Brébeuf. Les 300 ans qui la séparent du martyre du prêtre jésuite ne semblent pas avoir affecté la vivacité de son souvenir dans l'imaginaire de la jeune fille puisqu'elle le mentionne sans commentaire explicatif. Brébeuf, le personnage historique, a laissé une marque plus profonde dans l'imagination de Vanessa que la vie réelle de sa voisine et camarade de classe. Cependant, et malgré le gouffre historique et culturel séparant Vanessa du jésuite, la présence du martyr français dans l'imagination de la jeune fille est peut-être moins surprenante que l'on pourrait croire; comme nous le verrons, Jean de Brébeuf et son sort occupent une place complexe dans l'imaginaire canadien.

Bien évidemment, Brébeuf est d'abord connu grâce à ses activités missionnaires lors du contact entre les premiers colons français de la NouvelleFrance et les habitants du Nouveau Monde - rencontre culturelle formant le noyau de l'expérience historique canadienne et dont les effets se font encore ressentir aujourd'hui. Pourtant, au-delà de son importance historique incontestable, Brébeuf est visiblement beaucoup plus qu'un acteur clé de ce premier contact. Comme le suggère Guy Laflèche (1986), il est devenu une figure mythique, une personne dont l'importance ne réside pas seulement dans ses actions, mais bien dans la fonction idéo-culturelle que sa vie, et surtout sa mort, sont venues à représenter. Ce n'est pas en première instance sa vie et ses activités en Huronie au XVIIe siècle qui ont assuré à Brébeuf sa pérennité, mais la valeur

près la même chose. Ma connaissance des Indiens n'était pas du tout vaste. Je ne me souvenais pas avoir déjà vu un vrai Indien et ma prise de conscience que Piquette provenait des habitants de Big Bear et Poundmaker, de Tecumsah, d'Iroquois qui avaient dévoré le cœur du père Brébeuf - tout cela lui donna un attrait instantané à mes yeux ». Toutes les traductions de l'anglais vers le français sont de l'auteur. 
symbolique que l'on attribua à sa vie dans de nombreuses représentations depuis sa mort.

Ce processus de représentation, ainsi que la création d'un mythe qui en a découlé, s'est effectué (et continue d'être produit) selon les besoins de toute une gamme de discours, chacun ayant son but idéologique propre (de l'hagiographie de l'Église catholique canadienne s'appuyant sur une historiographie de la nation canadienne à la recherche d'une trame narrative triomphaliste de ses origines, jusqu'à une révision de cette même trame narrative des origines). Parmi ces modes de représentation clés encore peu étudiés se trouvent les traditions francophone et anglophone de la littérature canadienne. C'est spécifiquement à cette forme de représentation littéraire que le présent article s'intéressera, en se penchant sur le portrait de la vie et de la mort de Brébeuf dans des textes représentatifs de l'institution littéraire du Canada. Plus particulièrement, l'article étudiera la puissance symbolique de cette image dans le développement historique des significations mouvantes de l'imaginaire canadien. Ce que je souhaite démontrer est que la mort de Brébeuf peut être lue comme une représentation métonymique de trois itérations de la trame narrative de la nation canadienne en développement, et que chacune de ces représentations, à son tour, désigne une compréhension ponctuelle de l'imaginaire canadien selon une perspective provenant des trois nations fondatrices du pays ${ }^{2}$. En lisant les représentations littéraires de la mort historique de Brébeuf, nous retracerons également l'évolution de l'idée des origines de la culture nationale au Canada selon la perspective globale des nations canadiennes.

\footnotetext{
2 Ainsi, en approchant le legs culturel complexe de Brébeuf dans l'imaginaire canadien, mon but ne sera donc pas de récapituler les fonctions et l'importance qui lui ont été attribuées (ou à ses confrères missionnaires) dans les sphères religieuses et sociales depuis le milieu du XIXe siècle. Ce sujet important a déjà été traité par d'autres chercheurs comme Guy Laflèche dans les six volumes de sa série Les Saints Martyrs canadiens et, plus récemment, par Emma Anderson dans son livre The Death and Afterlife of North American Martyrs paru en 2013.
} 
Issus de ces trois regroupements à la fois historiques et nationaux, les trois textes de référence pour la discussion qui va suivre sont : le spectacle dramatique de 1930 de Jean Laramée, L'Âme huronne, qui marque la culmination d'une tradition d'hagiographie de l'Église catholique qui s'efforça de reprendre le martyre de Brébeuf afin de redynamiser la foi et la nation canadienne-française; le long poème en vers blanc d'E. J. Pratt de 1940 intitulé Brebeuf and His Brethren qui fournit une base historique pour le développement d'une expression mythique de la nation canadienne anglophone au milieu du XXe siècle; et, finalement, le roman contemporain de 2013 de l'écrivain controversé Joseph Boyden, The Orenda, qui revisite le moment historique du premier contact entre Européens et Autochtones afin de réviser les origines d'une relation interculturelle, ce qui implique, à la suite de la réinterprétation littéraire, un réajustement de l'imaginaire canadien.

\section{Brébeuf en Nouvelle-France}

Même si les aventures de Brébeuf dans les forêts du Canada n'ont jamais cessé d'être mises au service de la mythologie, les grandes lignes historiques de la vie de Jean de Brébeuf sont bien établies (Talbot 1949; Latourelle 1993, 2019). Brébeuf est né en Normandie en 1593. Il rejoint la Compagnie de Jésus, les Jésuites, en 1617, et devient prêtre en 1622. En 1625, il arrive en Nouvelle-France où il passe son premier hiver avec les Montagnais. L'été suivant, il voyage au pays des Hurons où il établit la première mission. Il reste en Huronie jusqu'en 1629, lors de la brève perte de la colonie aux mains des Anglais. Après un retour en France de 4 ans, il revient ensuite en Nouvelle-France en 1633. En 1634, il réussit à faire le long voyage de retour au pays des Hurons. Capturé par les Iroquois (Haudenosaunee) pendant leur guerre d'extermination contre les Hurons (Wendats), Brébeuf est torturé et tué le 16 mars 1649. Il est canonisé en 
1930 avec sept autres religieux, un groupe connu collectivement sous l'appellation « les Saints Martyrs canadiens ».

Mort en 1649, Brébeuf quitta aussitôt l'histoire du cours des évènements pour entrer dans le domaine de la représentation historique, un processus qui commença immédiatement par les descriptions que firent deux jésuites, Christophe Regnault et Paul Ragueneau, pour les Relations des jésuites. Déjà, dans la Relation de 1649 de Ragueneau, Brébeuf est couronné du titre "premier Apostre des Hurons ». En dépit de son importance dans les Relations dès sa mort, ce ne fut qu'au XIXe siècle qu'une confluence de conditions historiques et culturelles se produisit pour propulser Brébeuf dans le domaine social populaire, surtout au Québec. Timothy Pearson suggère que l'une des conséquences de la Rébellion des patriotes fut l'opportunité offerte à l'Église de participer à la création d'une mémoire collective pour la population française au-delà de la dominance politique et administrative anglophone (Pearson 2008: 333-336). Parallèlement à la succession au siège épiscopal de l'évêque Ignace Bourget en 1840, au retour au Canada en 1842 des Jésuites, à l'établissement des missions catholiques dans l'Ouest, à la publication des Relations des jésuites en 1858 (Gagnon 1978 : 37) et, en général, à l'expansion de l'ultramontanisme dans la société franco-canadienne, le discours de persévérance, sinon de survivance, représenté par Brébeuf et les martyrs canadiens, offrit aux membres de l'Église catholique un modèle d'autodéfinition culturelle fort utile pour une société en situation minoritaire. Guy Laflèche est encore plus explicite dans son identification du rôle joué par Brébeuf et les Saints Martyrs dans un projet civilisationnel franco-canadien : «... les Saints Martyrs canadiens vont constituer la pièce maîtresse de notre Épopée nationale : on passe ainsi de la piété religieuse à la dévotion nationale » (Laflèche 1988 : 241). Laflèche voit Brébeuf et les Saints Martyrs comme ayant été instrumentalisés en tant qu'incarnation des valeurs ultramontaines d'un catholicisme d'État et cela, en trois étapes idéologiques : 
Le sens premier du mythe des Saints Martyrs canadiens est de vider le nationalisme de tout contenu libéral, qu'il soit progressiste ou révolutionnaire, et de réaliser en conséquence l'identification de la nation et de la religion; l'épisode est placé au centre de l'Épopée mystique dont le commencement est l'origine religieuse de la nation et la fin, son messianisme évangélisateur et civilisateur en Amérique. Deuxièmement: ce providentialisme repose sur l'origine divine de l'autorité qui revient, par conséquent, à la classe cléricale, ce qui implique d'une part la soumission et l'obéissance de tous et d'autre part le dévouement et le désintéressement des clercs, dont la preuve éclatante est la figure du martyr. [...] Troisièmement : après la nation catholique et l'autorité cléricale, l'épisode met en scène les ennemis de Dieu et l'enfer. [...] Ces Iroquois, ce sont donc les ennemis de l'Église et de l'État, c'est-à-dire les ennemis de la classe cléricale, tous ceux qui menacent la nation de l'infernal chaos social (Laflèche 1988 : 335).

C'est dans ce contexte sociohistorique qu'une campagne à la fois nationaliste et religieuse fut lancée qui aboutira à la béatification des huit martyrs canadiens en 1925, puis à leur canonisation en 1930.

\section{Brébeuf et le cléricalisme franco-canadien}

Malgré l'importance mythique de Brébeuf dans l'imaginaire québécois, il n'y a que peu de textes littéraires consacrés à la représentation artistique de sa vie; il semblerait que la signification sacrée de Brébeuf le rende imperméable aux exigences (plus encore, à la profanation) de l'histoire et de l'interprétation, dimensions forcément impliquées dans toute représentation littéraire ${ }^{3}$. Publiés dans les années 1920 et 1930, les textes du premier des trois regroupements nationaux de la représentation littéraire de la mort de Brébeuf proviennent de la culture franco-canadienne de la fin du XIXe siècle et du début du XXe. Ici, le portrait dressé de Brébeuf est issu du cléricalisme, un mouvement né au sein de la société québécoise qui accorda un rôle prépondérant à l'Église catholique et

\footnotetext{
${ }^{3}$ Les œuvres littéraires portant sur la représentation de Brébeuf dans le contexte du cléricalisme québécois sont les suivantes: Laure Conan, À l'ouvre et à l'épreuve (1891); Jean Féron, Jean de Brébeuf (1928); Jean Laramée, L'Âme huronne, drame (1931 [1930]); et Antonio Poulin, Brébeuf. Drame historique en cinq actes avec chœurs (1931).
} 
pour lequel les activités des premiers missionnaires français représentaient « une épopée mystique », et plus particulièrement, assoyaient «[1]es origines religieuses de la civilisation canadienne» (Goyau 1934: 21). Le traitement littéraire des premiers missionnaires de cette période coïncida avec la canonisation des huit Saints Martyrs canadiens en 1930, aboutissement d'une mobilisation sociétale qui cherchait à montrer que les jésuites avaient jeté les bases historiques de la civilisation franco-canadienne. Les textes en question suggèrent que l'intérêt transitoire pour le traitement littéraire de la vie de Brébeuf consolida la conception mythique de Brébeuf célébrée par sa canonisation plutôt que l'exploration d'autres fins idéologiques qu'auraient pu signifier sa vie et sa mort.

Un des textes représentatifs de la présentation de Brébeuf comme figure fondatrice de «l'Épopée nationale » québécoise est la pièce de théâtre de Jean Laramée, L'Âme huronne. Drame historique en deux parties cinq actes avec chours et orchestre. Mise en scène en 1930 et publiée en 1931, l'œuvre de Laramée fut visiblement préparée pour commémorer la canonisation des martyrs. Même si la pièce témoigne d'un souci de respecter les critères esthétiques classiques, l'objectif primordial du texte est explicitement de mettre la représentation de l'histoire au service de la propagation de la foi. La pièce de théâtre hagiographique en cinq actes est divisée en deux parties : une première section illustrant « les ruses du Malin » et une deuxième présentant « les secours du bon Ange ». Les deux parties rendent explicites les antipodes idéologiques et culturels qui animent la tension dramatique : d'une part, les forces infernales du diable, avec ses alliés le « sorcier » huron et les Iroquois; d'autre part, les agents du divin, les convertis hurons (en particulier les enfants) et, surtout, Brébeuf. En fait, tel qu'indiqué à la fin de l'introduction qui accompagne la version imprimée de la pièce, c'est le conflit, le combat entre ces deux forces - avec sa résolution eschatologique inévitable - qui est le moteur du texte. 
La fonction didactique, voire catéchistique de la pièce, est renforcée par l'appareil critique qui accompagne le texte théâtral. En plus d'une introduction par l'auteur, qui fournit des pistes explicites d'interprétation, la version publiée du texte contient aussi un appendice de 59 pages de « sources et documents » qui contextualise la mission jésuite en Huronie et la vie des prêtres jésuites afin de consolider le message hagiographique de la pièce. Ces sources et documents proviennent principalement des Relations des Jésuites et servent à diriger l'attention vers la dimension historique du texte, malgré l'utilisation ouvertement tendancieuse de l'Histoire. Dans l'introduction, Laramée indique la façon par laquelle ses intentions artistiques furent conduites par l'histoire telle qu'elle a été racontée par les Relations :

C'est cette page hérö̈que de l'histoire canadienne qui nous a suggéré l'idée d'une synthèse complète des labeurs apostoliques de nos saints Martyrs en terre huronne. Nous mettons en appendice les documents qui ont guidé notre marche (Laramée 1931 : 6).

Il suggère même que la volonté de rester fidèle à l'histoire telle qu'elle a été livrée par la tradition apostolique prévaut sur les demandes esthétiques, par exemple l'observation de la règle des trois unités.

En fait, L'Âme huronne est une véritable «fiction de l'histoire » à entendre de deux manières différentes. Premièrement, le texte est ancré dans le moment historique de la mission en Huronie selon la représentation narrative de l'Histoire livrée par les Relations des Jésuites. Les cinq actes de la pièce sont explicitement situés dans cinq épisodes historiques allant de 1634 jusqu'à la destruction des missions par les Iroquois en 1649. Les deux parties du texte dramatisent aussi des crises historiques dans les relations entre les prêtres jésuites et les aborigènes Hurons-Wendats. Et de la même manière que dans les Relations, la résolution de chaque crise confirme non seulement la persévérance et la force de Brébeuf, mais aussi la grandeur de son service pour la volonté et la gloire de Dieu, en dépit de 
ses souffrances extrêmes, en premier lieu celles qui lui ont été infligées par ses ennemis et qui confirment encore plus fortement la légitimité de son message. Deuxièmement, au-delà de l'évocation des événements historiques, le texte entre encore plus profondément dans la représentation offerte par les Relations afin de scruter les motivations psychologiques de Brébeuf. Comme Laflèche le suggère pour les "saints martyrs canadiens » en général, le personnage Brébeuf est le produit de représentations textuelles historiques, une représentation qui a dans son ensemble l'intention de servir des fins idéologiques religieuses (Laflèche 1988 : 244). Au service de cette même idéologie, L'Âme huronne utilise et en même temps reproduit le portrait hérö̈que de Brébeuf. À l'apogée de la première section de la pièce, «Les Ruses du Malin », Brébeuf confronte le Diable qui lui présente la vision des tourments iroquois qui l'attendent. Au lieu de les éviter, Brébeuf les accepte à l'image du Christ pour assurer la grâce éternelle des Hurons :

Oui mon Sauveur Jésus, si dans votre bonté, Vous me faites un jour la grâce du martyre, Ici, je vous fais vœu de m'y laisser conduire. Et je prétends que désormais ce ne soit plus Pour moi chose licite, ô mon maître Jésus. De fuir l'occasion de mourir, de répandre Mon sang pour vous ... Bien plus, j'ose prétendre Qu'à la prière de mon sang, vous vous rendrez ; Qu'à ce gage infaillible, enfin vous céderez Et que vous sauverez ce grand peuple qui souffre, Ne permettant pas qu'il s'engouffre Dans l'éternel enfer ... Frappez, frappez, mon Dieu, Prenez tout mon sang ... mais ... pitié, grâce pour eux, Miséricorde pour mes frères! ... (Laramée 1931 : 60)

Dans L'Âme huronne, l'événement le plus significatif de sa vie et de sa réputation, sa mort, se produit en coulisses (en conformité avec la bienséance d'une pièce de théâtre respectable), même si le récit récapitule certains détails dérangeants de sa 
torture. Le texte présente la mort de Brébeuf comme l'apogée de la deuxième partie de la pièce «Les Secours de Bon Ange», une mort qui est loin d'être «tragique » en ce sens qu'elle signifierait la futilité et le désespoir. Au contraire, c'est la mort de Brébeuf qui garantit les conséquences « comiques » (divines) de la pièce, la conversion du Grand Chef huron et l'acceptation de s'exiler loin de la Huronie, lieu représentant un mode de vie qui doit être remplacé par un autre :

Je sens qu'en m'éloignant du bûcher et d'Échon [Brébeuf],

Je cours, guidé par lui, vers d'autres Robes-Noires;

Je sens qu'en m'enfuyant je cours à la victoire;

Je sens qu'à ma prière un plus puissant répond...

En route, mes enfants, avec nous Dieu chemine;

$\mathrm{Au}$ seul bruit de son nom mon âme s'illumine.

Comme il nous a aimés, prouvons-lui notre amour (Laramée 1931 : 109).

Après la canonisation des martyrs canadiens en 1930, culmination d'un projet visant à revaloriser l'idée de la force constitutive de l'Église catholique dans la vie sociale canadienne, et après la publication de plusieurs textes littéraires servant à l'époque à commémorer la canonisation, l'institution littéraire franco-canadienne sembla avoir épuisé son intérêt pour la vie et la mort de Brébeuf, une génération avant les bouleversements de la Révolution tranquille.

\section{Brébeuf et le nationalisme (littéraire) anglo-canadien de l'après-guerre}

Dix ans plus tard, les années 1939 et 1940 virent la publication de deux textes littéraires anglo-canadiens - les textes du deuxième regroupement évoqué - qui repensent l'histoire de la vie et de la mort de Brébeuf comme l'incarnation d'une force historique transcendante et implacable menant, à travers les «héros» jésuites, à la création épique de la civilisation canadienne de l'époque. Ici, Brébeuf figure au centre d'un poème épique destiné à être lu comme 
un récit mythique de la nation canadienne ${ }^{4}$. Reprenant le dernier mot dit par Brébeuf dans L'Âme huronne, "frères », le long poème canonique canadien de E. J. Pratt s'intitule Brébeuf and His Brethren. Comme L'Âme huronne, Brébeuf and His Brethren s'appuie largement sur les Relations des Jésuites, mais aussi sur The Jesuits in North America écrit par l'historien américain Francis Parkman, un livre ayant eu une influence immense aux États-Unis et au Canada anglais. Contrairement à Laramée, qui ciblait d'abord les fidèles de la foi catholique, Pratt utilisa l'histoire de Brébeuf et de ses frères hurons et jésuites pour formuler un récit mythique qui, pendant une génération et demie, fut lu comme une épopée de l'établissement de la présence euro-canadienne en Amérique du Nord. Brébeuf and His Brethren fut écrit au début de la Deuxième Guerre mondiale dans le contexte d'un nationalisme naissant qui, tout comme le roman contemporain de Hugh MacLennan, Two Solitudes, incorpora les expériences francophones et une connaissance des particularités de l'environnement et de l'histoire dans l'articulation d'une vision des origines de la nation. C'est dans cet esprit que le poème fut reçu au sein de la littérature canadienne. En 1946, par exemple, le premier critique anglo-canadien, Northrop Frye, dans son article « The Narrative Tradition in English-Canadian Poetry », chercha à décrire le processus par lequel les écrivains de la jeune institution littéraire canadienne essayèrent de développer une voix nationale-littéraire appropriée à la spécificité de l'expérience canadienne. Frye vit en Pratt et Brébeuf and His Brethren la réalisation la plus aboutie de cette démarche à la fois littéraire et nationale : «Brébeuf is not only the greatest but the most complete Canadian narrative, and brings together into a single

\footnotetext{
${ }^{4}$ Le deuxième texte de l'époque est de Franklin Davey McDowell, The Champlain Road (1939, gagnant du Prix du gouverneur général : romans et nouvelles de langue anglaise en 1939). Alors que The Champlain Road a été relégué à l'oubli par l'institution littéraire (anglo-)canadienne, Brébeuf and His Brethren a joui d'un statut canonique pendant plus de 30 années formatrices, à une époque clé dans l'établissement d'une littérature nationale anglo-canadienne.
} 
pattern all the themes we have been tracing» (Frye 1971: 153)5. La valeur accordée par Frye à Pratt comme médiateur de l'imaginaire canadien sera reprise et développée pendant le troisième quart du siècle.

Le poème composé de douze sections replace les expériences de Brébeuf et ses frères dans des perspectives beaucoup plus larges que le sort particulier de Brébeuf. Contrairement au Brébeuf des représentations francophones du début du siècle au Québec, le Brébeuf de Pratt n'incarne pas les seules valeurs de l'Église catholique dans la construction d'une civilisation canadienne religieuse. Pour Pratt, "[t]he story of the Jesuit missionaries to Canada is not only a great act in the national drama: it is a chapter in the history of religion: it is a saga of the human race » (Pratt 1983: 114) . Même s'il est encore animé par l'esprit de Dieu, le Brébeuf de Pratt participe au vaste projet de l'histoire, une entreprise qui dépasse les courants religieux sectaires. Le tout début du poème place Brébeuf dans le rôle d'un néophyte répondant à l'appel d'un Dieu qui se manifeste comme un « vent », une force motrice de l'Histoire destinée à changer le monde :

The winds of God were blowing over France,

Kindling the hearths and altars, changing vows

Of rote into an alphabet of flame.

The air was charged with song beyond the range

Of larks, with wings beyond the stretch of eagles.

...

The story of the frontier like a saga

Sang through the cells and cloisters of the nation,

\footnotetext{
${ }^{5}$ «Brébeuf est non seulement le récit canadien le plus important, mais aussi le plus complet, et il regroupe en un seul modèle tous les thèmes que nous avons tracés ". Toute une génération de l'après-guerre au Canada anglophone salua positivement Pratt et Brébeuf and His Brethren. Encore en 1973, Sandra Djwa put avancer avec assurance : " It is generally agreed that E. J . Pratt (1882-1964) is Canada's major poet and that Brébeuf and His Brethren (1940) his narrative of the Jesuit Mission to New-France, is the most significant poem yet written in Canada » («Il est généralement admis que E. J. Pratt (1882-1964) est le principal poète canadien et Brébeuf et ses frères (1940), son récit de la mission jésuite en Nouvelle-France, le plus important poème jamais écrit au Canada ») (Djwa 1973 : 82).

${ }^{6}$ « l'histoire des missionnaires jésuites au Canada n'est pas seulement un grand acte du drame national : c'est un chapitre de l'histoire de la religion: c'est une saga de la race humaine ».
} 
$\ldots$

And in Bayeux a neophyte while rapt

In contemplation saw a bleeding form

Falling beneath the instrument of death,

Rising under the quickening of the thongs,

Stumbling along the Via Dolorosa.

No play upon the fancy was this scene,

But the Real Presence to the naked sense (Pratt $1940: 1-3)^{7}$.

En répondant à sa vocation, Brébeuf accepte de quitter les conforts de la France pour entrer, héroïquement, au cœur d'un continent inconnu et menaçant. Le récit épique trace un voyage à travers le temps et l'espace qui est physique, mais aussi existentiel, et qui est voué à se terminer dans une mort prédite par les visions de Brébeuf, par les expériences d'autres jésuites martyrs et, surtout, par la connaissance historique du lecteur. Tout comme sa mort dans L'Âme huronne rappelle explicitement la mort du Christ, la mort de Brébeuf dans Brébeuf and His Brethren s'effectue à l'image de la crucifixion du Christ, « a bleeding figure ... stumbling along the Via Dolorosa $»^{8}$. Dès le début du poème, la trajectoire de sa présence au Canada est prédestinée à se terminer dans la mort; l'arc de la vie de Brébeuf est inscrit - tout comme dans L'Âme huronne - dans les marqueurs historiques de 1625 jusqu'à 1649 qui guident et dirigent le poème inexorablement vers une fin narrativement et historiquement inévitable.

Bien que le poème débute et s'achève avec une image religieuse, avec les interventions des critiques littéraires canadiens, son importance s'étend au-delà des significations exclusivement religieuses pour jouer un rôle fondateur dans

\footnotetext{
7 «Un vent divin soufflait sur la France, / attisant âtres et autels, transformant les prières / récitées sans conviction en serments enflammés. / L'air fut chargé avec un chant au-delà de l'envol / des alouettes, avec des ailes au-delà de l'étendue des aigles. [...] L'histoire de la frontière comme une saga, / résonnait à travers les cellules et les cloîtres du pays, [...] Et à Bayeux un néophyte plongé / dans la contemplation vit une forme sanglante / tomber sous l'instrument de la mort / se lever sous le supplice du fouet / et trébucher le long de la Via Dolorosa. / Loin d'être un spectre de l'imagination, cette scène / était une manifestation aux facultés nues de la Présence divine ».

8 «... une forme sanglante ... trébuchant le long de la Via Dolorosa ».
} 
l'établissement d'une conception puissante de l'imaginaire (anglo-) canadien de l'après-guerre. Pour Northrop Frye, par exemple, c'est surtout Pratt qui donna à une sensibilité canadienne sa forme littéraire. Plus particulièrement, Frye fut convaincu du pouvoir de Pratt de fournir une expression poétique des archétypes canadiens. Brébeuf and His Brethren occupe un rôle générateur dans le développement de sa fameuse théorie d'une "mentalité d'assiégés » (garrison mentality), selon laquelle l'identité canadienne est caractérisée par un sens de l'angoisse et un désir de s'installer dans un groupe protégé de l'environnement menaçant qui l'entoure. Dans la genèse de la formulation de sa théorie, lors d'un article de 1965, "Conclusion to a Literary History of Canada», Frye évoque directement le Pratt de Brébeuf and His Brethren:

A garrison is a closely knit and beleaguered society, and its moral and social values are unquestionable. In a perilous enterprise one does not discuss causes or motives: one is either a fighter or a deserter. Here again we may turn to Pratt, with his infallible instinct for what is central in the Canadian imagination. The societies in Pratt's poems are always tense and tight groups engaged in war, rescue, martyrdom, or crisis, and the moral values expressed are simply those of that group...

(Frye $1971: 226$, je souligne) ${ }^{9}$.

Pour Frye, ce fut le Brébeuf de Pratt qui personnifia le projet archétypal de l'établissement $\mathrm{du}$ « jardin » d'une civilisation canadienne dans « les bois » de la nature nord-américaine.

En 1972, à un moment clé du développement du nationalisme littéraire au Canada anglophone, Margaret Atwood renforça et vulgarisa l'idée de la mentalité d'assiégés dans Survival: A Thematic Guide to Canadian Literature, un

\footnotetext{
9 «Une garnison est une société à la fois étroitement tissée et assiégée, et ses valeurs morales et sociales sont incontestables. Dans un exercice périlleux, on ne discute pas de causes ni de motifs : on est soit un guerrier, soit un déserteur. Ici encore, nous pouvons nous tourner vers Pratt, avec son instinct infaillible pour ce qui est central dans l'imaginaire canadien. Les sociétés dans les poèmes de Pratt se présentent toujours comme des groupes tendus et hermétiques engagés dans la guerre, le sauvetage, le martyre, ou la crise, et les valeurs morales exprimées sont celles du groupe ».
} 
livre dans lequel elle identifie également Brébeuf and His Brethren comme étant « the all-Canadian poem » (Atwood 1972 : 93). Près de 30 ans après la parution de Brébeuf and His Brethren, ce poème épique, qui raconte le martyre d'un prêtre jésuite d'il y a 300 ans, se voit donc canonisé comme l'expression type d'une identité nationale cherchant à s'exprimer à travers les textes littéraires d'une tradition culturelle encore jeune.

L'idée de la mentalité d'assiégés donne à la nature comme force menaçante un rôle primordial. Dans Brébeuf and His Brethren, comme cela fut proclamé par Frye et Atwood, ce sont les autochtones, et surtout les Iroquois, qui représentent la forme humaine d'une nature féroce vis-à-vis de laquelle les colons cherchaient un abri en établissant une mission, une garnison dans laquelle ils vont établir une culture. Évidemment, nous, lecteurs d'aujourd'hui, avons bien raison de résister à une telle représentation des peuples autochtones qui les dépeint comme l'incarnation du mal ou qui les traite comme de simples rebuts de la trajectoire de l'esprit historique $^{10}$. Pourtant, et sans vouloir ignorer les implications troublantes de la représentation des autochtones dans le poème, il y a une autre lecture possible qui a l'avantage de contribuer à la ligne d'argumentation qui voit dans Brébeuf and His Brethren un texte expressif d'une sensibilité canadienne du misiècle. Dans Brébeuf and His Brethren, tout comme dans les écrits de Frye (encore une fois influencé par Pratt), les autochtones sont, à l'instar de Brébeuf, les acteurs d'un déroulement de l'histoire qui s'effectue au-delà de la volonté des individus. Frye rappela cette même idée dans un article de 1976 intitulé « Haunted

\footnotetext{
${ }^{10}$ Même si sa portée fut limitée, il y eut presque immédiatement une critique adressée à la représentation des peuples autochtones dans le poème de Pratt. Déjà, en 1941, le grand poète et contemporain de Pratt, F. R. Scott, rédigea son propre poème (une riposte à Pratt) avec le même titre «Brébeuf and His Brethren » : «When Lalemant and de Brébeuf, brave souls, / Were dying by the slow and dreadful coals / Their brother Jesuits in France and Spain / Were burning heretics with equal pain. / For both the human torture made a feast: / Then is priest savage, or Red Indian priest? » (Scott 1957 : 63). («Quand Lalemant et de Brébeuf, des âmes courageuses, / mouraient sous la lente et redoutable braise / leurs frères jésuites en France et en Espagne / brûlaient des hérétiques avec une douleur égale. / Pour les deux la torture humaine a fait un festin: / Alors le prêtre est-il sauvage, ou l'Indien rouge prêtre?)
} 
by Lack of Ghosts »: "... in Pratt's Brébeuf and His Brethren, the martyrdom of the Jesuit missionaries is nothing that they [les Iroquois] are seeking for itself, but it is the one real triumph of their service of Christ » (Frye $2003: 482)^{11}$. De ce point de vue, les autochtones sont précisément les frères de Brébeuf (tel qu'indiqué dans le titre, ses brethren); tout comme Brébeuf, ils répondent aux «winds of God» impersonnels à leur façon.

Dans le même article et en faisant référence à un autre poème canadien, Frye étendit l'argumentation en suggérant qu'au lieu d'être la négation du progrès historique, les autochtones sont essentiels à sa continuation à travers l'indigénisation des colonisateurs :

In this poem the Indians symbolize the primitive mythological imagination which is being reborn in us: in other words, the white Canadians, in their imagination, are no longer immigrants but are becoming indigenous, recreating the kind of attitudes appropriate to people who really belong here (Frye $2003: 487)^{12}$.

Cette représentation, inévitable ici, des peuples autochtones en tant qu'outils de l'acculturation des colonisateurs européens, demeure douteuse à plusieurs niveaux. Pourtant, et sans tenir compte de la légitimité finalement accordée à cette idée, le fait demeure que la mort de Brébeuf servit à l'élaboration d'une conception puissante, même si passagère, de l'identité canadienne.

\section{Brébeuf décolonisé}

Étant donné que tous les textes qui reprennent l'image de Brébeuf incluent une représentation au mieux caricaturale des peuples autochtones, il n'est pas

\footnotetext{
11 «Dans Brébeuf et ses frères de Pratt, le martyre des missionnaires jésuites n'est rien que [les Iroquois] cherchent en soi, mais il demeure le seul triomphe réel de leur service au Christ».

12 «Dans ce poème, les autochtones symbolisent l'imagination mythologique primitive qui est en voie de renaître en nous : autrement dit, les Canadiens blancs, dans leur imagination collective, ne sont plus des immigrants, mais ils sont en voie de devenir indigènes, recréant le genre d'attitudes appropriées aux gens qui sont ici véritablement à leur place ».
} 
étonnant que le groupe le plus récent - le troisième - s'étant intéressé à la vie et la mort de Brébeuf soit issu d'une littérature axée sur une perspective autochtone ${ }^{13}$. Ces représentations des missionnaires jésuites et de Brébeuf ont en commun d'offrir une lecture révisionniste à l'égard du contact historique entre missionnaires et autochtones. Bien que les textes n'aient pas été tous rédigés par des écrivains autochtones, ils se rejoignent généralement dans leur renversement d'une acceptation sans questionnement de la légitimité du projet des missionnaires européens, de manière à mieux représenter la perspective des peuples des Premières Nations face à l'arrivée des jésuites au pays des Hurons ${ }^{14}$. Ce changement de perspective assure le caractère révisionniste des textes et il s'exprime surtout par une représentation des jésuites fortement différente de celle du passé, même si les jésuites et leur arrivée au pays des Wendats continuent d'occuper le centre de l'intrigue littéraire. Brébeuf perd ici son rôle positif de pionnier dans l'établissement inévitable d'une civilisation franco-religieuse ou

\footnotetext{
${ }^{13}$ Les textes de référence de la troisième tranche littéraire qui sont le fruit d'un intérêt pour Brébeuf sont: Eldon Garment, Brebeuf: A Martyrdom of Jean De (1977); James W. Nichol, Sainte-Marie among the Hurons (1977); Brian Moore, Black Robe (1985); Daniel David Moses, Brébeuf's Ghost ([1996] 2000) et Joseph Boyden, The Orenda (2013). Le fait que l'émergence d'une représentation (progressivement plus) révisionniste des premiers missionnaires coïncide historiquement avec la présence croissante des voix autochtones dans l'institution littéraire canadienne est à noter.

${ }^{14}$ La problématique de l'appartenance nationale des auteurs qui souhaitent représenter l'expérience (historique) des peuples autochtones est soulevée explicitement avec Joseph Boyden, un écrivain qui fut initialement identifié et validé par l'institution littéraire canadienne en tant qu'écrivain autochtone. En 2016, trois ans après l'apparition de The Orenda, l'autoreprésentation de Boyden comme personne ayant un héritage autochtone fut contestée par Jorge Barrera de l'APTN National News. Pourtant, et en dépit de critiques ensuite exprimées à l'égard du roman et du romancier, il est incontestable que The Orenda fut reçu et loué dans la culture littéraire canadienne précisément comme la représentation d'une perspective indigène sur un moment historique canadien. Le commentaire de Kathryn Labelle, une historienne wendat, peut être lu comme représentatif de la réception initiale du roman: "Overall, The Orenda is a welcome contribution to a twenty-first century resurgence in Wendat studies. It is not only an award-winning source of fiction but also a useful tool to encourage people to take the next step and look into the history behind the story » (Labelle 2015 : 429). ("Dans l'ensemble, The Orenda est une contribution bienvenue à la résurgence des études wendat au XXI' siècle. Il s'agit non seulement d'une source de fiction primée, mais également d'un outil utile pour inciter les gens à franchir une nouvelle étape et à se pencher sur l'histoire qui se cache derrière l'histoire »). L'évaluation positive du roman ne fut pas partagée par Hayden King, qui l'a caractérisé d'« un écrit colonial et un alibi moral » (2014).
} 
anglo-canadienne. Loin d'être un héros, le Brébeuf des représentations des dernières décennies est la source des maux divers qui furent introduits dans le monde autochtone par les représentants des religions et de la culture européennes. En réimaginant la figure de Brébeuf, ces représentations proposent une vision nouvelle de l'histoire canadienne et, avec elle, une réévaluation de l'imaginaire canadien.

Dans The Orenda, par exemple, ce n'est plus la perspective culturelle des jésuites qui domine le récit; la narration est partagée entre trois protagonistes qui représentent les trois groupes centraux impliqués dans les événements: Christophe, un prêtre jésuite errant dans la Huronie; Snow Falls, une jeune fille iroquoienne capturée pour être mise en adoption chez les Wendats; et Bird, un guerrier Wendat qui se bat pour la survie de son peuple. Ce n'est plus seulement que la perspective européenne n'est plus privilégiée comme auparavant en tant que source «naturelle»d'autorité et de légitimité face à l'obscurantisme et l'ignorance des autochtones, elle est maintenant présentée comme étant chancelante, voire criminelle - source directe et indirecte de la catastrophe multidimensionnelle qu'ont vécue les Hurons-Wendats. N'étant plus la manifestation historique ni d'une présence providentielle dans la colonisation de la Nouvelle-France, ni des épreuves nécessaires pour la création de la jeune nation canadienne, Brébeuf devient la figure métaphorique d'une tragédie historique pour les peuples autochtones de l'Amérique du Nord dont on peut encore ressentir les effets aujourd'hui.

Mais même si l'importance de Brébeuf est radicalement réévaluée, le récit de sa vie décrit selon la tradition historique reste visible à travers le récit. The Orenda, comme les autres histoires de Brébeuf, reste redevable de l'historique livré par les Relations des Jésuites, mais en en inversant le sens. Donc, le prêtre représenté dans le roman ne porte pas le nom Jean de Brébeuf - il est connu sous 
le nom de Christophe (faisant écho à Christophe Colomb) ou, plus souvent, sous ceux de «corbeau» ou «Robe noire». Et loin d'être en plein contrôle de luimême, presque infaillible dans ses activités, le «Corbeau noir » de The Orenda est une figure de faiblesse, d'incompréhension et de malheur tragique pour les autochtones. Christophe n'est pas le véhicule héroïque d'une doctrine salvatrice, mais plutôt le « corbeau » qui « flew over the great water from their old world to perch tired and frightened in the branches of ours ${ }^{15}$, le vecteur d'un mot contagieux qui est voué à tuer l'orenda, la force magique animant la culture Huron-Wendat :

And when they cawed that our magic was unclean, we laughed, took a little offence, even killed a few of them and pulled their feathers for our hair. We lived on. But that word, unclean, that word, somehow, like an illness, like its own magic, it began to grow (Boyden $2013: 5)^{16}$.

Bref, toutes les caractéristiques positives attribuées à Brébeuf dans les Relations des Jésuites ou par la tradition subséquente sont renversées. Pourtant, en raison de la centralité des Relations pour la reconstruction de n'importe quelle narration des événements historiques - à des fins soit valorisantes, soit révisionnistes -, les traces des épreuves de Brébeuf perdurent, faisant de The Orenda un palimpseste plutôt qu'une réécriture de l’histoire.

Cette logique de la reconnaissance nécessaire d'un récit historique antérieur ressort avec grande évidence dans la représentation de l'expérience la plus significative de la vie de Brébeuf, sa mort. Dans The Orenda, la mort de Brébeuf n'est pas la preuve d'une présence providentielle dans les affaires historiques de la Huronie du XVIIe siècle, elle n'est même pas la culmination des événements de l'intrigue du roman ou une considération pour d'autres

\footnotetext{
15 «... a survolé la grande eau de leur ancien monde pour se percher fatigué et effrayé dans les branches de la nôtre ».

16 «Et quand ils ont croassé que notre magie était impure, nous avons ri, avons été quelque peu froissés, nous en avons même tué quelques-uns et arraché leurs plumes pour nos cheveux. Nous avons continué à vivre. Mais ce mot, impur, ce mot, comme une maladie en quelque sorte, comme sa propre magie, il a commencé à grandir ».
} 
protagonistes $\mathrm{du}$ roman. La mort par torture de Christophe présentée dans l'avant-dernier chapitre du roman est montrée à la première personne dans une perspective figurativement solipsiste. Les détails de sa mort, les formes spécifiques de la torture connues de la tradition dérivant des Relations, sont rappelés : c'est sans conteste la mort de Brébeuf telle qu'elle a été livrée par les livres d'histoire. Mais une fois contextualisée autour du sort du peuple HuronWendat qui est au centre du roman, la mort de Christophe-Brébeuf n'a plus aucune importance. Dans The Orenda, vidé de toute signification positive, Brébeuf est finalement mort et enterré, même si demeurent les conséquences négatives et durables de son intervention historique au pays des Wendats ${ }^{17}$.

À ma connaissance, The Orenda est le dernier texte littéraire canadien à retravailler l'histoire de Jean de Brébeuf, mais la trajectoire historique de l'institution littéraire canadienne ne nous donne aucune raison de penser que la vie de Brébeuf, et surtout sa mort, aient finalement atteint le terme de leur représentation fictionnelle au Canada. Nous avons vu que la vie historique de Brébeuf fut source d'inspiration pour trois regroupements de textes littéraires qui ont adapté sa vie à travers la formation de trois discours sociohistoriques différents faisant de Jean de Brébeuf un initiateur de «fictions de l’histoire ».

\footnotetext{
${ }_{17}$ Dans la pièce de théâtre Brébeuf's Ghost de Daniel David Moses, le renversement de l'importance de Brébeuf est encore plus explicite. Brébeuf est dépourvu de toute qualité de meneur et de toute force vitale. Toute l'action se déroule dans les mois après sa mort; il fait figure dans la pièce d'un fantôme maléfique entouré de mouches et d'asticots, un spectre de la mort qui rend cannibales tous ceux qui entrent en contact avec lui.
} 


\section{Bibliographie}

Anderson, E. (2013). The Death and Afterlife of North American Martyrs. Cambridge, Harvard University Press.

Atwood, M. (1972). Survival. Toronto, House of Anansi.

Boyden, J. (2014). Dans le grand cercle du monde. Traduction par Michel Lederer de The Orenda. Paris, Albin Michel.

Boyden, J. (2013). The Orenda. Toronto, Penguin Books.

Campeau, L. (1987). La mission des Jésuites chez les Hurons, 1634-1650. Montréal, Éditions Bellarmin.

Carleton, S. (2014). " The Complexities of Colonialism : An Alternative Reading of The Orenda ». Canadian Dimension, vol. 48, n 3, p. 43.

Conan, L. (1943 [1891]). À l'œuvre et à l'épreuve. Montréal, Beauchemin.

Djwa, S. (1973). «The Civil Polish of the Horn: E. J. Pratt's "Brébeuf and his Brethren" ». Ariel, vol. 4, n³, p. 82-102.

Féron, J. (1928). Jean de Brébeuf : roman canadien inédit. Montréal, E. Garand.

Frye, N. (2003 [1976]). « Haunted by Lack of Ghosts ». Dans J. O'Grady et D. Staines (dir.), Northrop Frye on Canada. tome 12, Collected Works of Northrop Frye. Toronto, University of Toronto Press, p. 472-92.

Frye, N. (1971). The Bush Garden. Toronto, House of Anansi.

Frye, N. (dir). (1962). Collected Poems of E.J. Pratt. Toronto, Macmillan.

Gagnon, S. (1978). Le Québec et ses historiens de 1840 à 1920. Québec, Les Presses de l’Université Laval.

Garnet, E. (1977). Brebeuf: A Martyrdom of Jean De. Erin, Press Porcépic.

Goyau, G. (1934). Une épopée mystique. Les origines religieuses du Canada. Paris, Éditions Spes.

Greer, A. (dir.). (2000). The Jesuit Relations: Natives and Missionaries in SeventeenthCentury North America. Boston, Bedford/St. Martin's. 
King, H. (2014). « The Orenda Faces Tough Criticism from First Nations Scholar ». $C B C$ News, 7 mars; www.cbc.ca/news/indigenous/the-orenda-faces-toughcriticism-from-first-nations-scholar-1.2562786 (consulté le 3 mars 2019).

Labelle, K. (2015). «Compte rendu de The Orenda». Canadian Historical Review, vol. $96, n^{\circ} 3$, p. 426-29.

Laflèche, G. (1986). «La spiritualité des Jésuites de la Nouvelle-France: L'interprétation des Visions de Jean de Brébeuf (1640)». Voix et images, vol. $11, n^{\circ} 3$, p. $464-474$.

Latourelle, R. (1993). Jean de Brébeuf. Montréal, Bellarmin.

Latourelle, R. (s. d.) «Brébeuf, Jean de». Dictionnaire biographique du Canada; http://www.biographi.ca/fr/bio/brebeuf_jean_de_1F.html (consulté le 3 mars 2019).

Laurence, M. (1993 [1970]). "The Loons». Dans A Bird in the House. Chicago, University of Chicago Press, p. 108-120.

Laurence, M. (1990). Les Saints Martyrs canadiens. tome 3, Le martyre de Jean de Brébeuf selon Paul Ragueneau. Laval, Les Éditions du Singulier.

Laurence, M. (1988). Les Saints Martyrs canadiens. tome 1, Histoire du mythe. Laval, Les Éditions du Singulier.

Laramée, J. (1931 [1930]). L'Âme huronne. Drame. Montréal, L'Action paroissiale.

Lemire, M. (1970). Les grands thèmes nationalistes du roman historique canadienfrançais. Québec, Presses de l’Université Laval.

Madsen, K. (2013). «Compte rendu de The Orenda». Northern Review, vol. 37, p. 215-18.

McDowell, F. D. (1939). The Champlain Road. Toronto, Macmillan.

Moore, B. (1985). Black Robe. New York, Dutton.

Moses, D. D. (2000 [1976]). Brébeuf's Ghost: A Tale of Horror in Three Acts. Toronto, Exile Editions. 
Nichol, J. W. (1977). Sainte-Marie among the Hurons: A Play. Toronto, Playwrights Co-op.

Parkman, F. (1922 [1867]). The Jesuits in North America in the Seventeenth Century. Boston, Little, Brown, and Company.

Pearson, T. G. (2008). «Becoming Holy in Early Canada: Performance and the Making of Holy Persons in Society and Culture». Thèse de doctorat, Montréal, McGill University.

Poulin, A. (1930). Brébeuf. Drame historique en cinq actes avec chœurs. Montréal, Messager canadien.

Pratt, E. J. (1983). E. J. Pratt on His Life and Poetry. Susan Gingell (dir.). Toronto, University of Toronto Press.

Pratt, E. J. (1940). Brébeuf and His Brethren. Toronto, Macmillan.

Scott, F. R. (1957). Eye of the Needle: Satire, Sorties, Sundries. Montréal, Contact Press.

Talbot, F. X. (1956 [1949]). Saint among the Hurons: The Life of Jean de Brébeuf. New York, Image Books. 